\title{
Hydrogen Sensor Based on High-Birefringence Fiber Loop Mirror with Sol-Gel Pd/WO 3 Coating
}

\author{
Ya-nan Zhang ${ }^{1,2, *}$, Huijie Peng ${ }^{1}$, Tianmin Zhou ${ }^{1}$, Lebin Zhang ${ }^{1}$, Yuyan Zhang ${ }^{3}$, Yong Zhao ${ }^{1,2}$ \\ ${ }^{1}$ College of Information Science and Engineering, Northeastern University, Shenyang \\ 110819, China \\ ${ }^{2}$ State Key Laboratory of Synthetical Automation for Process Industries, Shenyang 110819, \\ China \\ ${ }^{3}$ Shenyang Institute of Engineering, Shenyang 110136, China \\ *Corresponding author: zhangyanan@ise.neu.edu.cn
}

\begin{abstract}
A hydrogen sensor based on high-birefringence fiber loop mirror (HBFLM) with sol-gel $\mathrm{Pd} / \mathrm{WO}_{3}$ coating was demonstrated. The sensing structure was formed by inserting a section of polarization maintaining fiber $(\mathrm{PMF})$ with $\mathrm{Pd} / \mathrm{WO}_{3}$ coating in fiber loop mirror. The $\mathrm{Pd} / \mathrm{WO}_{3}$ coating was prepared by sol-gel method and coated on PMF by dip-coating method, which is simple fabrication, low cost, and has good binding force with fiber. When the hydrogen concentration around $\mathrm{Pd} / \mathrm{WO}_{3}$ coating was changed, it would induce the strain change of polarization maintaining fiber, and then shift the interference spectrum of HBFLM. Therefore, the hydrogen concentration can be measured by monitoring the wavelength shift of the interference spectrum. Experimental results showed that the resonance wavelength had a blue shift with the increase of hydrogen concentration and the total shift of the resonance wavelength was $\sim 2.18 \mathrm{~nm}$ within the concentration range of $0-1 \%$. The sensor had simple structure, low cost, high sensitivity, good repeatability, and well stability.
\end{abstract}

Keywords: Optical fiber sensor; Hydrogen sensor; High-birefringence fiber loop mirror; $\mathrm{Pd} / \mathrm{WO}_{3}$. 


\section{Introduction}

Hydrogen is a clean and reusable energy source, which has been widely used in various fields such as automobile industry, aerospace engineering, chemical processing, and so on. However, hydrogen is also extremely easy to leak out of containers and even explode in air due to its high diffusion coefficient $\left(0.16 \mathrm{~cm}^{2} / \mathrm{s}\right.$ in air), low ignition energy $(0.018 \mathrm{~mJ})$, high combustion heat (285.8 $\mathrm{kJ} / \mathrm{mol})$, and wide explosion concentration range (4\% 75\%). Therefore, it is necessary to carry out warning when hydrogen concentration is one-tenth of the lower explosion limit, namely, $\underline{0.4 \%}$. Besides, it is meaningful to monitor the variation tendency of hydrogen concentration at the range of $0-1 \%$ and give an effective guidance for taking precautions.

Due to the advantages of optical fiber sensor, such as small size, immune to electromagnetic interference, intrinsic safety, and remote sensing, optical fiber hydrogen sensor has been proposed early in 1999 [1], which is based on evanescent field principle [2]. Although such sensor is the most common one in practical application, the hydrogen concentration is deduced by monitoring the transmitted intensity of light source, which is easily interrupted by external disturbances. Besides, this core-exposed method to generate evanescent field may weak the strength of sensing head, and then influence the mechanical stability of hydrogen sensor. Recently, with the further development of optical fiber technology, optical fiber hydrogen sensors have progressed rapidly and attracted great attentions. Many wavelength-demodulated optical fiber hydrogen sensors have been demonstrated, such as fiber Bragg grating (FBG), Surface plasmon resonance (SPR), and interferometer. For the FBG based hydrogen sensor [3, 4], it present the particular advantages of distributed measurement and small size, but its sensitivity is still low even some special techniques, such as side-polished, chemical etching, and tapering, are used [5-7]. For the SPR based hydrogen sensor, it receives continuously growing attentions due to its advantages of high sensitivity and rapid detection [8-10], but the production requirement and cost of the metallic film are relatively high. On the other hand, hydrogen sensors based on optical fiber interferometers have been much more popular, which include Mach-Zechnder interferometer [11-13], Fabry-Perot interferometer [14-16], and Sagnac interferometer [17-19]. Compared to other sensors, optical fiber interferometers have high sensitivity and flexible structure. However, the interference spectrum of hydrogen sensor based on Mach-Zehnder interferometer is usually confusing due to 
multiple modes interfering with each other [11-13]. Hydrogen sensor based on Fabry-Perot interferometer has drawbacks in practical application, such as restriction on cavity size owing to coupling loss, large transmission loss, and offset of fiber end faces [14-16]. In contrast, hydrogen sensor based on Sagnac interferometer, also known as fiber loop mirror (FLM), has more simple structure and is easy to fabricate $[17,18]$. In 2013, Y. Kim et al. demonstrated an optical fiber Sagnac interferometer hydrogen sensor with a Pd-coated PMF [17]. At a hydrogen concentration of $4 \%$, the interference spectrum showed a wavelength shift of $\sim 2.48 \mathrm{~nm}$. But the coating of pure

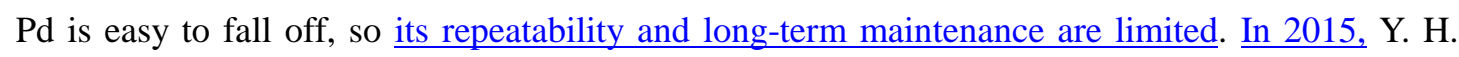
Yang et al. demonstrated another fiber Sagnac interferometer hydrogen sensor based on a polarization-maintaining photonic crystal fiber with $\mathrm{Pd} / \mathrm{Ag}$ composite film [18]. The shift of interference spectrum was $\sim 1.310 \mathrm{~nm}$ within the hydrogen concentration range of $0-1 \%$ and the sensor sensitivity was $\sim 131 \mathrm{pm} / \%$ within $1-4 \%$ hydrogen concentration. But the cost of polarization-maintaining photonic crystal fiber and the fabrication cost of $\mathrm{Pd} / \mathrm{Ag}$ film are all high.

In this paper, a hydrogen sensor based on high-birefringence fiber loop mirror (HBFLM) with $\mathrm{Pd} / \mathrm{WO}_{3}$ coating was proposed. The $\mathrm{Pd} / \mathrm{WO}_{3}$ fabricated by sol-gel method acted as the sensitive material to the ambient hydrogen. Compared to pure Pd film, the binding force between the film and fiber can be increased; while compared to pure $\mathrm{WO}_{3}$ film, the hydrogen selectivity of sensor can be enhanced. The reason is due to the difference of the reaction mechanisms: 1) the Pd in

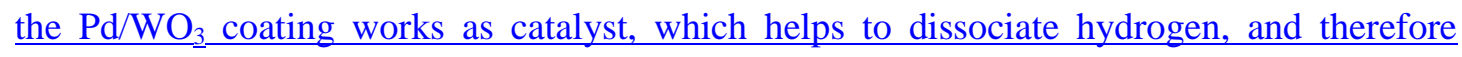
promote the chemical reaction between $\mathrm{WO}_{3}$ and hydrogen; 2) while in case of $\mathrm{Pd}$ layer, the sensing mechanism is based on phase transition of $\mathrm{PdH} x$. Since $\alpha$ and $\beta$ phase of $\mathrm{PdH} x$ have quite different structure, mechanical mismatch during the phase transition cycle will generate dislocations in the coating, and finally cracks arise [19]. Besides, the influence of other interfering gases to the sensitivity of our proposed hydrogen sensor is very small and can be neglected [20,21].

By coating $\mathrm{Pd} / \mathrm{WO}_{3}$ film on polarization maintaining fiber (PMF), the interference spectrum of HBFLM will be shifted with hydrogen concentration, due to the strain change of PMF. As a result, the hydrogen concentration can be measured by monitoring the wavelength shift of the interference spectrum at constant temperature of $20^{\circ} \mathrm{C}$ and constant humidity of $40 \%$. In this case, 
the experimental results demonstrated that the sensitivity of the hydrogen sensor can reach $-2.18 \mathrm{~nm} / \% \underline{\left(R^{2}=0.968\right)}$ within the hydrogen concentration range of $0-1 \%$.

\section{Operation Principle}

Fig. 1 shows the structural configuration of the HBFLM, which consists of a 3-dB optical coupler (OC) and a section PMF. Both ends of the PMF are spliced to conventional single-mode fiber (SMF). The input light is split into two beams propagating clockwise and counterclockwise by the $3 \mathrm{~dB}$ coupler. Then the two beams are recombined at the coupler and interference appears due to the birefringence property of the inserted PMF. The transmission spectrum of the HBFLM can be described as [22]:

$$
T(\lambda)=\left[\sin \left(\theta_{1}+\theta_{2}\right) \cos \left(\frac{\pi B L}{\lambda}\right)\right]^{2}
$$

where $\theta_{1}$ and $\theta_{2}$ are the angles between the light at both ends of the PMF and the fast or slow axis of the PMF, respectively, and $B, L$ and $\lambda$ are the birefringence, the length of PMF and the wavelength, respectively. The wavelength spacing between the transmission dips can be written as [22, 23]:

$$
\Delta \lambda \approx \frac{\lambda^{2}}{B L}
$$

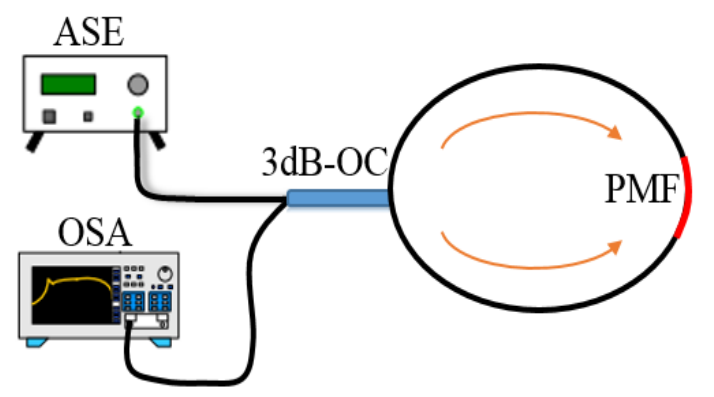

Fig. 1. Structural configuration of the HBFLM.

Fig. 2 shows the interference spectra of the HBFLM for different $B$ and $L$ of the PMF. It can be observed that when other parameters are fixed, the birefringence $B$ of the PMF decreases as the period of the transmission spectrum increases, and the length L of the PMF is inversely proportional to the period of the transmission spectrum. When a strain is applied to the HBFLM, the birefringence and the fiber length of the PMF will change, and the resonance wavelengths will shift. 


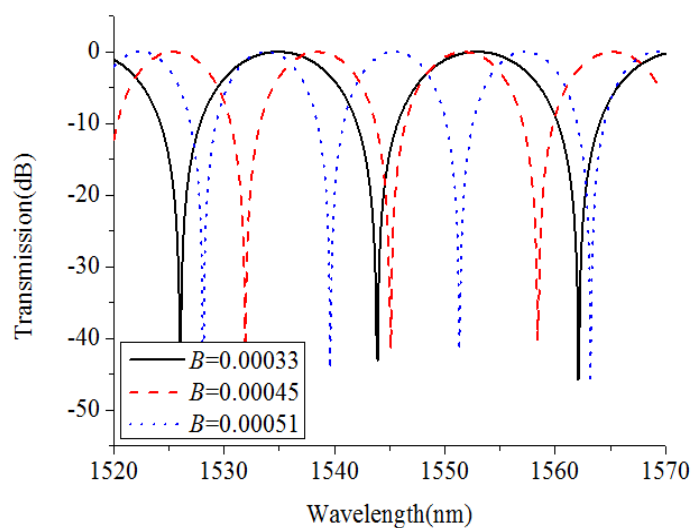

(a)

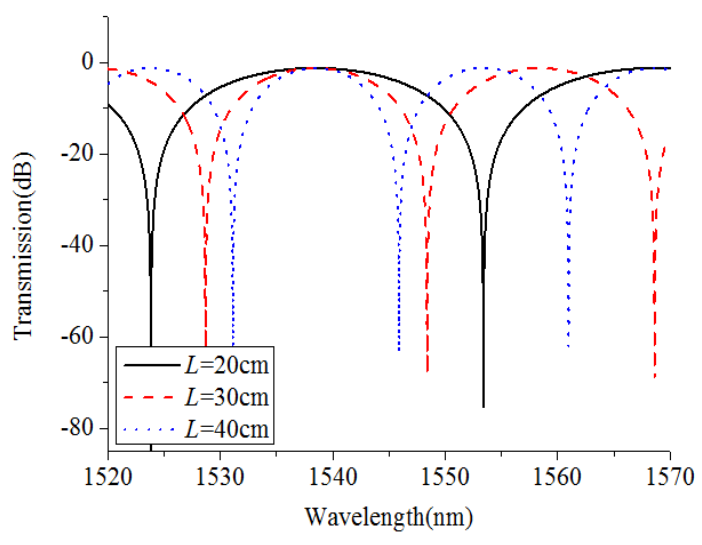

(b)

Fig. 2. Interference spectra of the HBFLM for different birefringence and lengths of the PMF.

The metal Pd has high hydrogen absorption capacity and high selectivity to hydrogen, while the $\mathrm{WO}_{3}$ film has better hydrogen sensitivity, good adhesion and mechanical properties. When $\underline{\mathrm{Pd} / \mathrm{WO}_{3}} \underline{3}_{\text {contacts with hydrogen, the absorption and decomposition of hydrogen takes place under }}$ $\underline{\text { the catalysis of Pd. Hydrogen atoms are transferred to the surface of } \mathrm{WO}_{3} \text { by } \mathrm{Pd} \text { and then spread }}$ along the surface of the inner holes of $\mathrm{WO}_{3}$, which cause the expansion of the $\mathrm{Pd} / \mathrm{WO}_{3}$ coating and imposing strain on the PMF coated $\mathrm{Pd} / \mathrm{WO}_{3}$ and associate with the change of hydrogen concentration. Therefore, hydrogen concentration can be measured by monitoring the wavelength shift of interference spectrum of the HBFLM, and the interference spectrum has a blue shift.

\section{Experimental Setup}

Fig. 3 shows the experimental configuration of the proposed hydrogen sensor, which consists of an amplified spontaneous emission source (ASE), an optical spectrum (OSA) with spectral resolution of $0.02 \mathrm{~nm}$, and a HBFLM. The HBFLM consists of a 3-dB optical coupler (OC) with low insertion loss, a PMF with length of $15 \mathrm{~cm}$ and $\mathrm{Pd} / \mathrm{WO}_{3}$ coating. A tank of pure nitrogen and a tank of nitrogen and hydrogen mixture with standard 3.5\% hydrogen concentration were prepared for hydrogen sensing experiments. First, the vacuum pump was used to remove the residual gas in the pipeline. Then, opening the valves. Here, the gases from the tanks were controlled by the gas-flow meters and then mixed into the measurement chamber. Finally, the combination of gases formed a stable atmosphere of hydrogen with certain concentration and the sensing structure perceived the change of the hydrogen concentration in the chamber. 


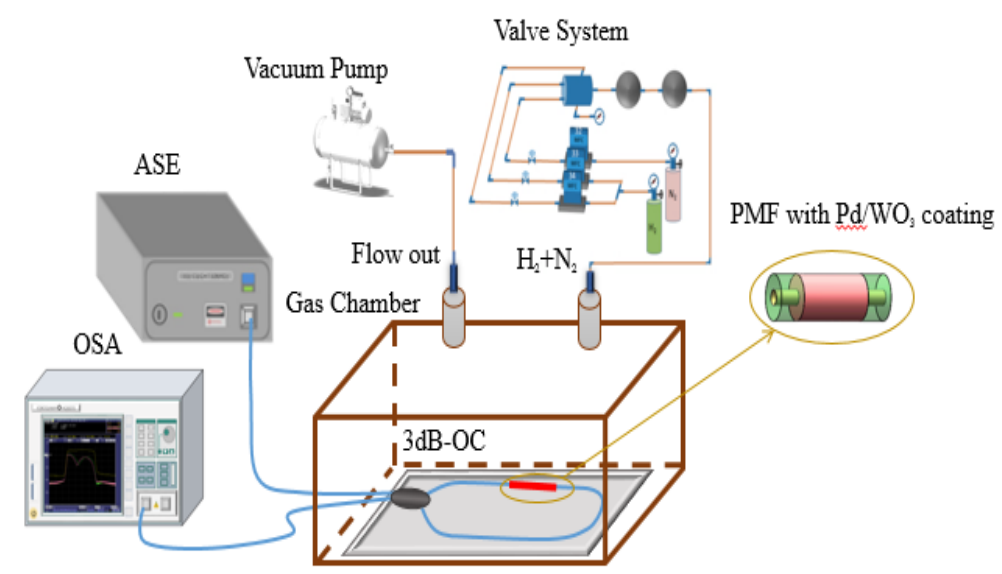

Fig. 3. Experimental configuration of the $\mathrm{HBFLM}$ with $\mathrm{Pd} / \mathrm{WO}_{3}$ coating.

The hydrogen sensitive material in our sensor is $\mathrm{Pd} / \mathrm{WO}_{3}$, which can be prepared by various methods $[24,25]$. In this paper, the sol-gel method is chosen [26] due to its low cost and simple fabrication. The specific preparation process is shown in Fig. 4: (1) Chemical reactions between tungsten powder and hydrogen peroxide; (2) Removing of redundant tungsten powder and other impurities by filtering process, through which we can obtain the polymers tungstate solution, as shown in Fig, 5(a); (3) Removing of some insoluble impurities ; (4) Adding alcoholic solution to the above solution, along with the evaporation process, to obtain $\mathrm{WO}_{3}$ sol, as shown in Fig. 5(b); (5) Doping $\mathrm{PdCl}_{2}$ to $\mathrm{WO}_{3}$ sol to obtain the $\mathrm{Pd} / \mathrm{WO}_{3}$ sol, as shown in Fig. 5(c); (6) Coating the $\mathrm{Pd} / \mathrm{WO}_{3}$ sol to the PMF surface, by using the dip coating machine; (7) Improving the performance of the film and enhancing and binding force between the film and fiber by conducting a thermal treatment under temperature of $100^{\circ} \mathrm{C}$ for $1 \mathrm{~h}$. After the above steps, a brownish-yellow sol with homogeneous stabilization and a PMF coated with smooth film are obtained. During the experiment, several samples with different doping ratios were prepared. With the increase of the doping amount, the viscosity of the sol increased and more ethanol was needed to dissolve, which indicated that the structure of the sol was more unstable and had a tendency to rapidly solidify. The quality and stability of the samples with different doping ratios and the quality of the coating were evaluated. As a result, for doping ratio below 1:100, the coating quality is good. But at the same time, the high amount of doping was helpful to improve the response time and selectivity of hydrogen sensor. Therefore, we chose $1: 100$ as the optimum molar ratio of Pd:W in this manuscript by synthetically considering the coating quality and sensor properties. 


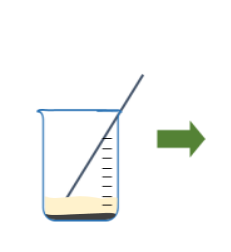

(1) Reaction

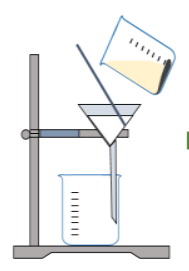

(2) Filter

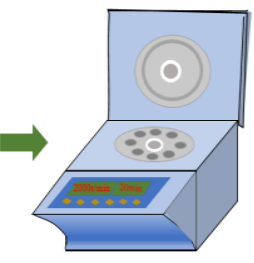

(3) Centrifuge

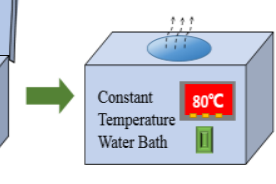

(4) Evaporation

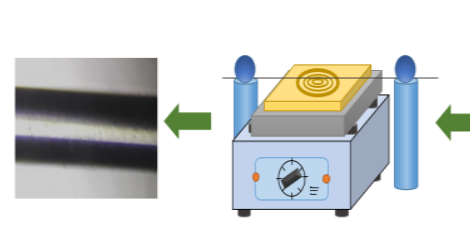

(8) Coating effect

(7) Thermal treatment

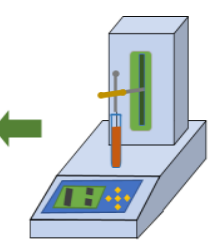

(6) Dip-coating

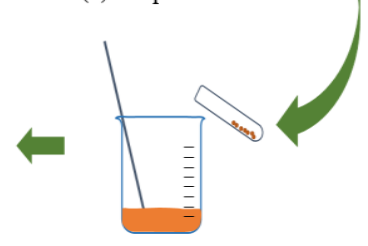

(5) Doping

Fig. 4. Preparation process of $\mathrm{Pd} / \mathrm{WO} 3$ coating.

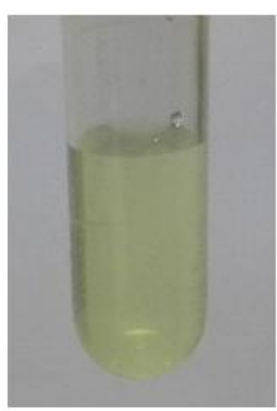

(a)

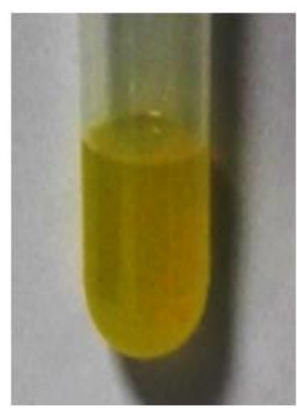

(b)

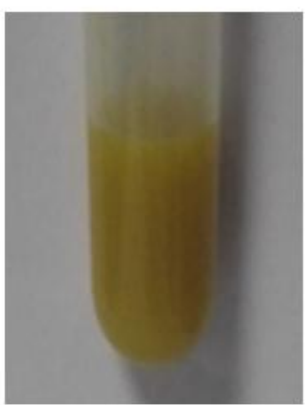

(c)

Fig. 5. Intermediate reaction solutions in the fabrication process of Pd/WO3.

During experiments, the chamber was purged with pure nitrogen ( $0 \%$ hydrogen concentration) before filled with hydrogen of different concentrations. Then gas with standard hydrogen concentrations of $0.2 \%, 0.4 \%, 0.6 \%, 0.8 \%$ and $1 \%$ was flowed into the chamber. The resonance wavelength of the interference spectrum was recorded when the spectrum became stable. $\underline{\text { It }}$ usually takes $\sim 1$ min. The main reason is that in our experiment the hydrogen concentration is controlled by mixing nitrogen and certain hydrogen with constant concentration of $3.5 \%$ as mentioned above, and the mixed hydrogen with specific concentration is gradually transmitted to $\underline{\text { sensor probe and the hydrogen concentration around the sensor probe is gradually varied. In }}$ practical test, this time delay can be largely decreased.

\section{Results and Discussions}

Fig. 6(a) exhibits interference spectra of the $\mathrm{HBFLM}$ with $\mathrm{Pd} / \mathrm{WO}_{3}$ coating under different 
hydrogen concentrations. It is observed that the resonance wavelength has a blue shift with the increase of hydrogen concentration and the total shift of the resonance wavelength is $\sim 2.18 \mathrm{~nm}$. Fig. 6(b) gives the tendency of the resonance wavelength versus hydrogen concentration. The good polynomial fit shows that the sensor has a high hydrogen sensitivity, each resonance wavelength shifts averaging $\sim 2.18 \mathrm{~nm} / \%$ in the concentration range from $0-1 \%$. The error bars for the six data points in Fig. 6(b) have been showed in Fig. 6(c). The output result (wavelength) of hydrogen sensor for each hydrogen concentration is change in a certain range, which is mainly due to the interferences of surrounding temperature, humidity, vibration, and the concentration error of measured gas. Compared to Ref. [17, 18], the proposed sensor was low cost, simple fabrication, and has a relatively high sensitivity.

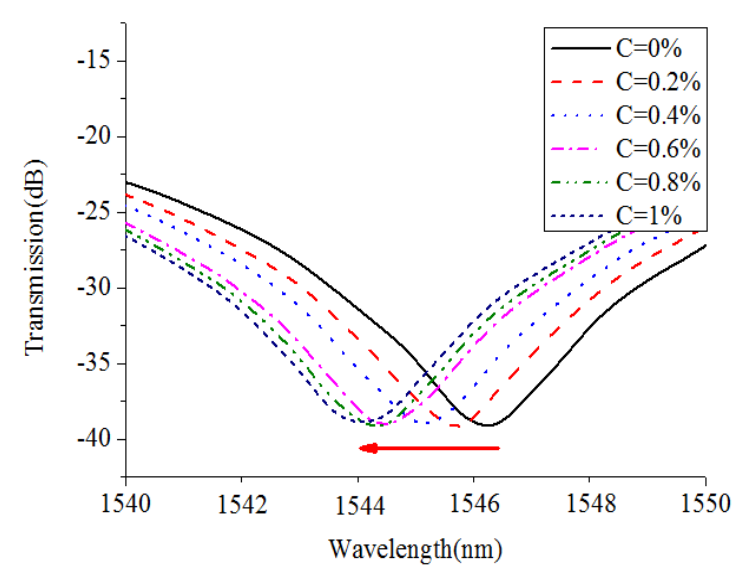

(a)

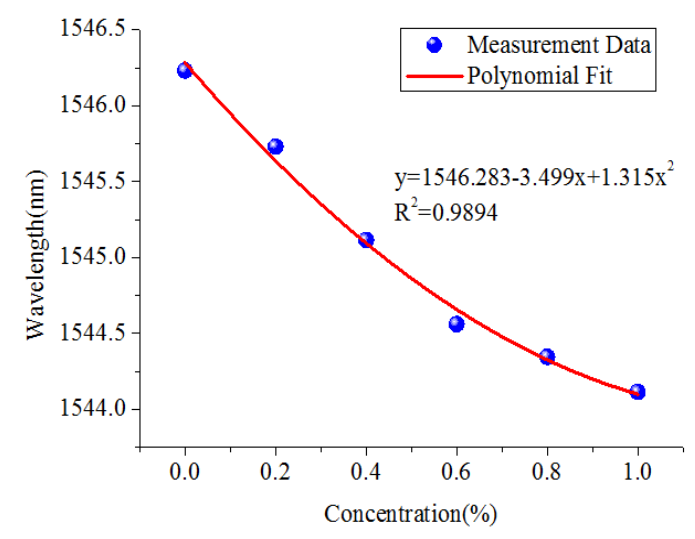

(b)

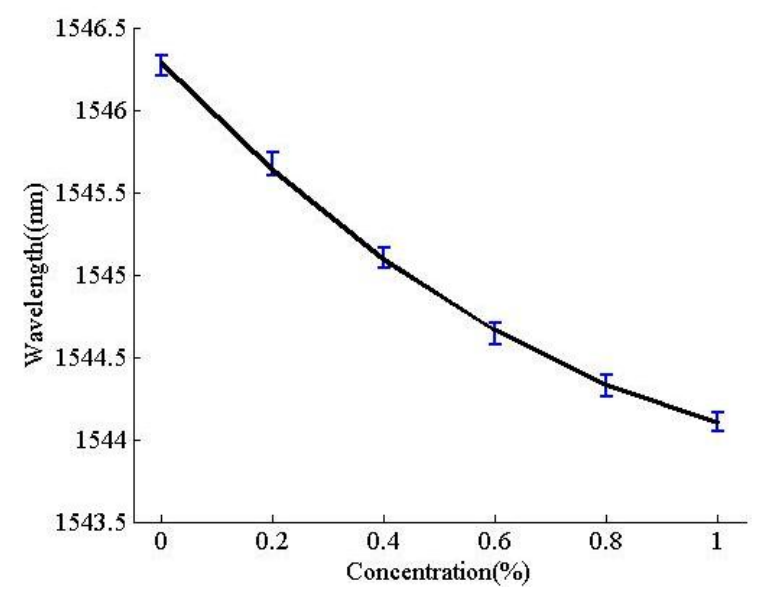

(c)

Fig. 6. (a) Interference spectrum of the HBFLM with Pd/WO3 coating under different hydrogen concentration.

(b) Relation between hydrogen concentration and the resonance wavelength. (c) Error variations of six data points. 
It should be mentioned that the sensitivity decreases as the hydrogen concentration increases which may be due to the hydrogen absorption of $\mathrm{Pd} / \mathrm{WO}_{3}$ reach to saturation when the hydrogen concentration is too high or ambient temperature changes. On the other hand, it is possible that the effective refractive index of the hydrogen sensitive material decreases as the hydrogen concentration increases, and this change will cause the resonance wavelength of the HBFLM move to the short wavelength direction. This result not only improves the sensitivity of the sensor, but also causes the nonlinear of sensor. In the future, to enhance the sensitivity, stability, and linearity of hydrogen sensor, we will try another materials, such as $\mathrm{SiO}_{2}, \mathrm{WO}_{3}, \mathrm{Fe}_{2} \underline{\mathrm{O}}_{3}, \mathrm{ZnO}$, $\underline{\mathrm{SnO}_{2}}, \mathrm{Pt}_{\mathrm{Al}} \mathrm{Al}_{\underline{3}}, \mathrm{O}_{3}$ and so on, in place of $\mathrm{WO}_{\underline{3}}$ [27].

In our experiment, the repeatability of the sensor is shown in Fig. 7(a). We repeated the hydrogen sensing experiment three times. There are some slight measuring errors in each test, which mainly result from the room temperature fluctuation and the measuring errors of optical instruments. The stability of the sensor is also shown in Fig. 7(b). At $0.4 \%$ hydrogen concentration, we recorded the shift of the resonance wavelength in 30 minutes with an interval of 5 minutes. The maximum error is $0.0021 \mathrm{~nm}$, which equivalents to the wavelength shift caused by $0.096 \%$ hydrogen concentration variation. Therefore, the small fluctuate can be neglected. Besides, in our experiment, the resolution of OSA is $0.02 \mathrm{~nm}$, so this fluctuate may be generated by the reading error. In a whole, the experimental results demonstrated that the sensor had high sensitivity, good

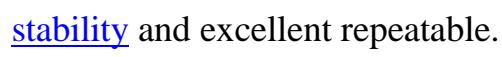

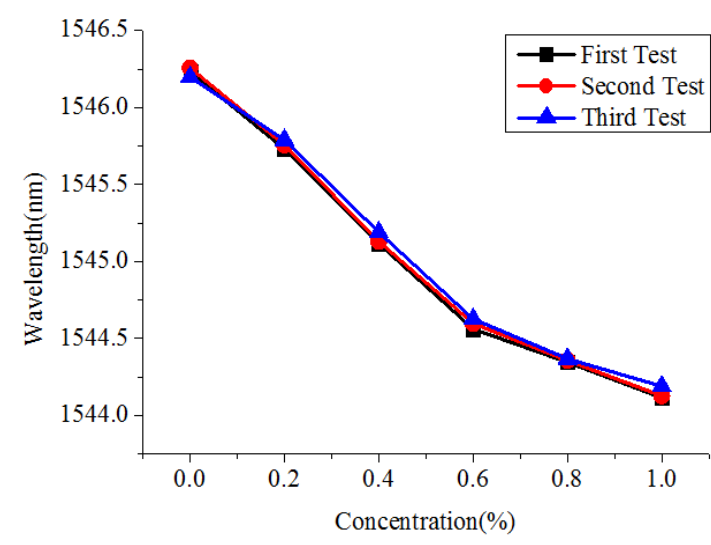

(a)

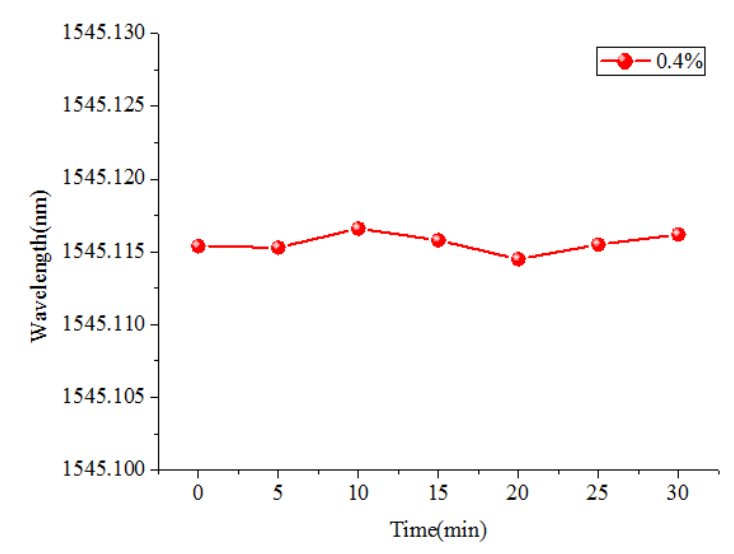

(b)

Fig. 7. (a) Repeatability of the sensor. (b) Resonance wavelength versus time. The concentration of the tested hydrogen is $0.4 \%$. 
The response time of the proposed sensor mainly depends on the sensitive material. In our previous manuscript, we have demonstrated that the response time of $\mathrm{Pd} / \mathrm{WO}_{3}$ based hydrogen sensor is no more than $33 \mathrm{~min}$ [28], so we have not repeated the experiments to measure the response time of the proposed sensor. What should be mentioned is that the response time may be improved by UV-light irradiation [29], doping graphene quantum dots [30], which will be carried out in our future work.

\section{Conclusion}

In conclusion, a hydrogen sensor with high sensitivity was demonstrated both in theory and experiment. When hydrogen concentration around the $\mathrm{PMF}$ with $\mathrm{Pd} / \mathrm{WO}_{3}$ coating was changed, it would induce the strain change of PMF, and then shift the interference spectrum of HBFLM. Therefore, the hydrogen concentration could be measured by monitoring the wavelength shift of interference spectrum. The resonance wavelength had a blue shift with the increase of hydrogen concentration. The total shift of the resonance wavelength was $\sim 2.18 \mathrm{~nm}$ within the concentration range from 0 to $1 \%$. The sensor had a number of valuable advantages, such as high sensitivity, excellent repeatability and stability, good safe performance, simple structure, low lost, suitable for practical application, and so on.

\section{Acknowledgments}

This work was supported in part by the National Science Foundation for Distinguished Young Scholars of China under Grant 61425003, the National Natural Science Foundation of China under Grant 61273059 and 61371200, the Fundamental Research Funds for the Central Universities under Grant N160404012 and N150401001, Liaoning Province Dr. started fund under Grant 201501087, and State Key Laboratory of Synthetical Automation for Process Industries under Grant 2013ZCX09. 


\section{References:}

[1] M. Tabib-Azar, B. Sutapun, R. Petrick, A. Kazemi. Highly sensitive hydrogen sensors using palladium coated fiber optics with exposed cores and evanescent field interactions. Sensors and Actuators B: Chemical, 1999, 56(1-2): 158-163.

[2] M. H. Yang, H. L. Liu, D. S. Zhang, X. L. Tong. Hydrogen sensing performance comparison of Pd layer and $\mathrm{Pd} / \mathrm{WO}_{3}$ composite thin film coated on side-polished single - and multimode fibers. Sensors and Actuators B: Chemical, 2010, 149(1): 161-164.

[3] J. X. Dai, M. H. Yang, Z. Yang, Z. Li, Y. Wang, G. P. Wang, Y. Zhang, Z. Zhuang, Performance of fiber Bragg grating hydrogen sensor coated with Pt-loaded $\mathrm{WO}_{3}$ coating, Sensors and Actuators B: Chemical, 2014, 190: 657-663.

[4] M. Buric, T. Chen, M. Maklad, P. R. Swinehart, and K. P. Chen, Multiplexable low-temperature fiber Bragg grating hydrogen sensors, IEEE Photonics Technology Letters, 2009, 21(21): 1594-1596.

[5] J. Jiang, G. M. Ma, C. R. Li, H. T. Song, Y. T. Luo, H. B. Wang. Highly sensitive dissolved hydrogen sensor based on side-polished fiber Bragg grating. IEEE Photonics Technology Letters, 2015, 27(13): 1453-1456.

[6] J. X. Dai, M. H. Yang, X. Yu, K. Cao, J. S. Liao. Greatly etched fiber Bragg grating hydrogen sensor with $\mathrm{Pd} / \mathrm{Ni}$ composite film as sensing material. Sensors and Actuators B: Chemical, 2012, 174: 253-257.

[7] S. Silva, L. Coelho, J. M. Almeida, O. Frazão, J. L. Santos, F. X. Malcata, M. Becker, M. Rothhardt, H. Bartelt. $\mathrm{H}_{2}$ sensing based on a Pd-coated tapered-FBG fabricated by DUV femtosecond laser technique. IEEE Photonics Technology Letters, 2013, 25(4): 401-403.

[8] A. Hosoki, M. Nishiyama, H. Igawa, A. Seki, K. Watanabe. A hydrogen curing effect on surface plasmon resonance fiber optic hydrogen sensors using an annealed $\mathrm{Au} / \mathrm{Ta}_{2} \mathrm{O}_{5} / \mathrm{Pd}$ multi-layers film. Optics Express, 2014, 22(15): 18556-18563.

[9] C. Perrotton, R. J. Westerwaal, N. Javahiraly, M. Slaman, H. Schreuders, B. Dam, P. Meyrueis. A reliable, sensitive and fast optical fiber hydrogen sensor based on surface plasmon resonance. Optics Express, 2013, 21(1): 382-390. 
[10] R. Tabassum, B. D. Gupta. Fiber optic hydrogen gas sensor utilizing surface plasmon resonance and native defects of zinc oxide by palladium. Journal of Optics, 2016, 18: 015004.

[11] M. Wang, M. H. Yang, J. Cheng, J. X. Dai, M. W. Yang, D. N. Wang. Femtosecond laser fabricated micro Mach-Zehnder interferometer with Pd film as sensing materials for hydrogen sensing. Optics Letters, 2012, 37(11): 1940-1942.

[12] F. X. Gu, G. Q. Wu, H. P. Zeng. Hybrid photon-plasmon Mach-Zehnder interferometers for highly sensitive hydrogen sensing. Nanoscale, 2015, 7, 924-929.

[13] Z. P. Yu, L. Jin, L. P. Sun, J. Li, Y. Ran, B. O. Guan. Highly sensitive fiber taper interferometric hydrogen sensors. IEEE Photonics Journal, 2016, 8(1): 6800309.

[14] M. Wang, M. H. Yang, J. Cheng, G. L. Zhang, C. R. Liao, D. N. Wang. Fabry-Pérot interferometer sensor fabricated by femtosecond laser for hydrogen sensing. IEEE Photonics Technology Letters, 2013, 25(8): 713-716.

[15] C. B. Yu, Li Liu, X. X. Chen, Q. F. Liu, Y. Gong. Fiber-optic Fabry-Perot hydrogen sensor coated with Pd-Y film. Photonics Sensors, 2015, 5(2): 142-145.

[16] Y. Wang, M. H. Yang, G. L. Zhang, J. X. Dai, Y. Zhang, Z. Zhuang, W. B. Hu. Fiber optic hydrogen sensor based on Fabry-Perot interferometer coated with sol-gel $\mathrm{Pt} / \mathrm{WO}_{3}$ coating. Journal of Lightwave Technology, 2015, 33(12): 2530-2534.

[17] Y. Kim, T. K. Noh, Y. W. Lee, E. S. Kim, B. S. Shin, S. M. Lee. Fiber-optic hydrogen sensor based on polarization-diversity loop interferometer. Journal of the Korean Physical Society, 2013, 62(4): 575-580.

[18] Y. H. Yang, F. L. Yang, H. Wang, W. Yang, W. Jin. Temperature-insensitive hydrogen sensor with polarization-maintaining photonic crystal fiber-based Sagnac interferometer. Journal of Lightwave Technology, 2015, 33(12): 2566-2571.

[19]_M. H. Yang, Y. Sun, D. S. Zhang, D. S. Jiang. Using $\mathrm{Pd} / \mathrm{WO}_{3}$ composite thin films as sensing materials for optical fiber hydrogen sensors. Sensor and Actuators B: Chemical. 2010, 143: 750-753.

[20]_M. Yang, H. L. Liu, D. S. Zhang, X. L. Tong. Hydrogen sensing performance comparison of $\underline{\mathrm{Pd} \text { layer and } \mathrm{Pd} / \mathrm{WO}_{3} \text { composite thin film coated on side-polished single- and multimode }}$ fibers. Sensors and Actuators B: Chemical. 2010, 149: 161-164. 
[21] J. Kukkola. M. Mohl, A. R. Leino, J. Maklin, N. Halonen, A. Shchukarev, Z. Konya, H. $\underline{\text { Jantunen, K. Kordas. Room temperature hydrogen sensors based on metal decorated } \mathrm{WO}_{3}}$ nanowires. Sensors and Actuators B: Chemical. 2013, 186: 90-95.

[22]_D. P. Zhou, L. Wei, W. K. Liu, J. W. Y. Lit. Simultaneous measurement of strain and temperature based on a fiber Bragg grating combined with a high-birefingence fiber loop mirror. Optics Communications, 2008, 281: 4640-4643.

[23] J. L. Chu, C. Y. Shen, F. Qian, C. Zhong, X. Zou. Simultaneous measurement of strain and temperature based on a long-period grating with a polarization maintaining fiber in a loop mirror. Optical Fiber Technology, 2014, 20: 44-47.

[24] J. X. Dai, M. H. Yang, Y. Chen, K. Cao, J. S. Liao. Hydrogen sensor based on D-shaped fiber Bragg grating coating coated with $\mathrm{WO}_{3}-\mathrm{Pd}$ composite films. Acta Photonica Sinica, 2011, 40(7): 1003-1006.

[25] M. H. Yang, Z. Yang, J. X. Dai, D. S. Zhang. Fiber optic hydrogen sensors with sol-gel $\mathrm{WO}_{3}$ coatings. Sensors and Actuators B: Chemical, 2012, 16-167: 632-636.

[26] S. Okazakia, H. Nakagawab, S. Asakuraa, Y. Tomiuchic, N. Tsujic, H. Murayamad, M. Washiya, Sensing characteristics of an optical fiber sensor for hydrogen leak. Sensors and Actuators B: Chemical, 2003, 93: 142-147.

[27] S. Masuzawa, S. Okazaki, Y. Maru, T. Mizutani. Catalyst-type-an optical fiber sensor for hydrogen leakage based on fiber Bragg gratings. Sensors and Actuators B: Chemical, 2015 , 217: 151-157.

[28]_Y. N. Zhang, Q. L. Wu, H. J. Peng, Y. Zhao. Photonic crystal fiber modal interferometer with $\mathrm{Pd} / \mathrm{WO}_{3}$ coating for real-time monitoring of dissolved hydrogen concentration in transformer oil. Review of Scientific Instruments, 2016, 87: 125002.

[29] X. X. Zhong, M. H. Yang, C. J. Huang, G. P. Wang, J. X. Dai, W. Bai. Water photolysis $\underline{\text { effect on the long-term stability of fiber optic hydrogen sensor with } \mathrm{Pt} / \mathrm{WO}_{3}}$. Scientific Reports, 2016, 6: 39160.

[30]_S. Fardindoost, A. I. Zad, Z. S. Hosseini, S. Hatamie. Detecting hydrogen using graphene quantum dots $/ \mathrm{WO}_{3} \underline{3}$ thin films. Materials Research Express, 2016, 3(11), 116407. 